DOI: https://doi.org/10.15688/jvolsu1.2016.6.10

UDC 517.547

LBC 22.161 .5

\title{
COMPLEX RIGIDITY OF TEICHMÜLLER SPACES
}

\author{
Samuel L. Krushkal
}

Doctor of Physical and Mathematical Sciences, Professor, Department of Mathematics, Bar-Ilan University, Ramat-Gan, 5290002, Israel, University of Virginia, Charlottesville, VA 22904-4137, USA slk6z@cms.mail.virginia.edu

Abstract. We outline old and new results concerning the well-known problems in the Teichmüller space theory, i.e., whether these spaces are starlike in the Bers holomorphic embedding and whether any Teichmüller space of dimension greater than 1 is biholomorhically equivalent to bounded convex domain in a complex Banach space.

Key words: Teichmüller spaces, holomorphic embeddings, Schwarzian derivative, convex domain, starlike, holomorphic section, conformally rigid domain, uniformly convex Banach space.

In the memory of Igor Zhuravlev

\section{Introductory remarks}

It is well-known that the Teichmüller spaces with their canonical complex structure are pseudo-convex. Moreover, all finite dimensional Teichmüller spaces are Runge domains, hence polynomially convex.

The folowing two longstanding problems relate to geometric convexity of these spaces. 1. For an arbitrary finitely or infinitely generated Fuchsian group $\Gamma$, is the Bers embedding of its Teichmüller space $\mathbf{T}(\Gamma)$ starlike?

2. Is any finite or infinite dimensional Teichmüller space of dimension greater than 1 biholomorphically equivalent to bounded convex domain in a complex Banach space $X$ (of the same dimension as $\mathrm{T}(\Gamma))$ ?

The first problem was stated among other open problems on Teichmüller spaces and Kleinian groups in the book [3] of 1974, collected by Abikoff.

The second problem was posed for the finite dimensional spaces by Royden and for the universal Teichmüller space by Sullivan. It relates to Tukia's result [17] which explicitly yields a real analytic homeomorphism of the universal Teichmüller space $\mathbf{T}=\mathbf{T}(\mathbf{1})$ onto a convex domain in a real Banach space.

The aim of this paper is to outline old and recent results obtained in solving these problems. 


\section{Teichmüller spaces are not starlike}

1. First recall that the Bers embedding represents the space $\mathbf{T}(\Gamma)$ as a bounded domain formed by the Schwarzian derivatives

$$
S_{w}=\left(\frac{w^{\prime \prime}}{w^{\prime}}\right)^{\prime}-\frac{1}{2}\left(\frac{w^{\prime \prime}}{w^{\prime}}\right)^{2}
$$

of holomorphic univalent functions $w(z)$ in the lower half-plane $U^{*}=\{z: \Im z<0\}$ (or in the disk) admitting quasiconformal extensions to the Riemann sphere $\widehat{\mathbb{C}}=\mathbb{C} \cup\{\infty\}$ compatible with the group $\Gamma$ acting on $U^{*}$.

It was shown in [10] that the universal Teichmüller space $\mathbf{T}=\mathbf{T}(\mathbf{1})$ has points which cannot be joined to a distinguished point even by curves of a considerably general form, in particular, by polygonal lines with the same finite number of rectilinear segments. The proof relies on the existence of conformally rigid domains established by Thurston in [15] (see also [2]).

This implies, in particular, that the universal Teichmüller space is not starlike with respect to any of its points, and there exist points $\varphi \in \mathbf{T}$ for which the line interval $\{t \varphi: 0<t<1\}$ contains the points from $\mathbf{B} \backslash \mathbf{S}$, where $\mathbf{B}=\mathbf{B}\left(U^{*}\right)$ is the Banach space of hyperbolically bounded holomorphic functions in the half-plane $U^{*}$ with norm

$$
\|\varphi\|_{\mathbf{B}}=4 \sup _{U^{*}} y^{2}|\varphi(z)|
$$

and $\mathbf{S}$ denotes the set of all Schwarzian derivatives of univalent functions on $U^{*}$. All $\varphi$ with finite norm (1) determine holomorphic functions on $U^{*}$ (as solutions of the Schwarz differential equation $S_{w}=\varphi$ ) which are only locally univalent.

Toki [16] extended the result on the nonstarlikeness of the space $\mathbf{T}$ to Teichmüller spaces of Riemann surfaces that contain hyperbolic disks of arbitrary large radius, in particular, for the spaces corresponding to Fuchsian groups of second kind. The crucial point in the proof of [16] is the same as in [10].

On the other hand, it was established in [12] that also all finite dimensional Teichmüller spaces $\mathbf{T}(\Gamma)$ of high enough dimensions are not starlike. It seems likely that this property must hold for all Teichmüller spaces of dimension at least two.

The non-starlikeness causes obstructions to some problems in the Teichmüller space theory and its applications to geometric complex analysis.

2. There is also a simpler proof that the universal Teichmüller space is not starlike. This proof, given recently in [8], provides explicitly the functions which violate this property. Its underlying geometric features are completely different and involve the Abikoff - Bers Zhuravlev theorem which yields that the domain $\mathbf{T}$ has a common boundary with its complementary domain in the space $\mathbf{B}$ (see [1], [4], [18]).

It is technically more convenient to deal here with univalent functions in the upper half-plane $U=\{z=x+i y: y>0\}$ denoting by $\mathbf{B}$ the corresponding space $\mathbf{B}(U)$ of hyperbolically bounded holomorphic functions in $U$.

Let $P_{n}$ be a convex rectilinear polygon with the finite vertices $A_{1}, A_{2}, \ldots, A_{n}$, and let the interior angle at the vertex $A_{j}$ be equal to $\pi \alpha_{j}$; then $0<\alpha_{j}<1$ and $\sum_{j=1}^{n} \alpha_{j}=n-2$.

The conformal map of $U$ onto $P_{n}$ is represented by the Schwarz - Christoffel integral

$$
f_{*}(z)=d_{1} \int_{0}^{z}\left(\xi-a_{1}\right)^{\alpha_{1}-1}\left(\xi-a_{2}\right)^{\alpha_{2}-1} \ldots\left(\xi-a_{n}\right)^{\alpha_{n}-1} d \xi+d_{0},
$$


where $a_{j}=f_{*}^{-1}\left(A_{j}\right) \in \mathbb{R}, a_{1}<a_{2}<\cdots<a_{n}$, and $d_{0}, d_{1}$ are the complex constants. Its logarithmic derivative $b_{f}=f^{\prime \prime} / f^{\prime}$ is of the form

$$
b_{f_{*}}(z)=\sum_{1}^{n}\left(\alpha_{j}-1\right) /\left(z-a_{j}\right)
$$

and its Schwarzian

$$
S_{f_{*}}(z)=\sum_{1}^{n} \frac{C_{j}}{\left(z-a_{j}\right)^{2}}-\sum_{j, l=1}^{n} \frac{C_{j l}}{\left(z-a_{j}\right)\left(z-a_{l}\right)},
$$

where

$$
C_{j}=\alpha_{j}-1-\frac{1}{2}\left(\alpha_{j}-1\right)^{2}<0, \quad C_{j l}=\left(\alpha_{j}-1\right)\left(\alpha_{l}-1\right)>0
$$

We normalize $f_{*}$ letting $a_{1}=0, a_{2}=1$ and fixing $a_{n}<\infty$; then $f_{*}(\infty)$ is an inner point of the edge $A_{n} A_{1}$.

Since the boundary $\partial P_{n}$ is a quasicircle, the function (2) admits a quasiconformal extension onto the lower half-plane $U^{*}$, hence $S_{f_{*}} \in \mathbf{T}$.

Denote by $r_{0}$ the positive root of the equation

$$
\frac{1}{2}\left[\sum_{1}^{n}\left(\alpha_{j}-1\right)^{2}+\sum_{j, l=1}^{n}\left(\alpha_{j}-1\right)\left(\alpha_{l}-1\right)\right] r^{2}-\sum_{1}^{n}\left(\alpha_{j}-1\right) r-2=0
$$

and let

$$
S_{f_{*}, t}=t b_{f_{*}}-\frac{t^{2}}{2} b_{f_{*}}^{2}, \quad t>0 .
$$

Theorem 1. For any convex polygon $P_{n}$, the Schwarzians $r S_{f_{*}, r_{0}}$ and $S_{f_{*}, r}$ with $0<r<r_{0}$ define the univalent on $U$ functions, and the corresponding harmonic Beltrami coefficients $v_{r}(z)=-(r / 2) y^{2} S_{f_{*}, r_{0}}(\bar{z})$ and $v_{r}(z)=-(1 / 2) y^{2} S_{f_{*}, r}(\bar{z})$ of their quasiconformal extensions to the lower half-plane $U^{*}$ are extremal (have minimal $L_{\infty}$-norm). Hence, for some $r$ between $r_{0}$ and 1 , the Schwarzians $r S_{f_{*}, r_{0}}$ and $S_{f_{*}, r}$ are the outer points of $\mathbf{T}$.

Note that for $r<r_{0}$ the solutions $w_{r}$ of each equation

$$
\left.w^{\prime \prime} / w^{\prime}\right)^{\prime}-\left(w^{\prime \prime} / w^{\prime}\right)^{2} / 2=\varphi_{r}(z), \quad z \in U
$$

with $\varphi_{r}=r S_{f_{*}, r_{0}}$ and $\varphi_{r}=S_{f_{*}, r}$ map $U$ conformally onto the quasidisks (either bounded or not), which can be regarded as the analytic polygons with vertices $w_{r}\left(a_{1}\right), \ldots, w_{r}\left(a_{n}\right)$, whose boundary consists either of $n$ real analytic arcs with nonzero intersection angles or else of arcs of spirals, which are analytic in their interior points.

This theorem yields, in particular, that any such $w_{r}$ does not admit extremal quasiconformal extensions of Teichmüller type.

The coefficients $v_{r}$ define the Ahlfors - Weill quasiconformal extension of $w_{r}$ to the lower half-plane $U^{*}$, and

$$
\left\|v_{r}\right\|_{\infty}=\frac{1}{2}\left\|\varphi_{r}\right\|_{\mathbf{B}}<1
$$

(provided that $\left\|\varphi_{r}\right\|_{\mathbf{B}}<2$ ).

The proof of Theorem 1 reveals an interesting connection between harmonic Beltrami coefficients and the Grunsky coefficient inequalities (first established in [11]). 
3. Note that non-starlikeness of the universal Teichmüller space is in fact the main step in the proof of most of the results mentioned in the beginning of this paper. By appropriate approximation, this property was extended to the spaces $\mathbf{T}(\Gamma)$ of sufficiently large dimensions. So Theorem 1 has the same corollaries. For example, the arguments in its proof provide simultaneously non-starlikeness of the space $\mathbf{T}$ in Becker's holomorphic embedding which represents this space as a bounded domain in the Banach space of holomorphic functions $\psi$ in the disk $\Delta^{*}=\{|z|>1\}$ with norm $\|\psi\|=\sup _{\Delta^{*}}\left(|z|^{2}-1\right) z \psi(z) \mid$. The points of this domain are the logarithmic derivatives $\psi_{f}=f^{\prime \prime} / f^{\prime}$ of univalent functions $f(z)=z+b_{0}+b_{1} z^{-1}+\ldots$ in $\Delta^{*}$.

4. As an example, consider the rectangles $P_{4}$. For any rectangle, all $\alpha_{j}=1 / 2$, hence the equation (4) assumes the form

$$
\frac{5}{4} r^{2}+2 r-2=0
$$

Its positive root $r_{0}=0.6966 \ldots$

\section{The second problem}

1. For a long time, the result of Tukia mentioned in the introduction remained the only known fact connecting Teichmüller spaces with geometric convexity. Recently it was established in [7] that the universal Teichmüller space $\mathbf{T}$ cannot be mapped biholomorphically onto a bounded convex domain in a uniformly convex Banach space, in particular, onto a convex domain in the Hilbert space. This yields a restricted negative answer to Sullivan's question.

The uniform convexity of a Banach space $X$ means strong convexity of its unit ball; namely, for any $x_{n}, y_{n}$ satisfying $\left\|x_{n}\right\| \leq 1,\left\|y_{n}\right\| \leq 1,\left\|x_{n}+y_{n}\right\| \rightarrow 2$ must be $\left\|x_{n}-y_{n}\right\| \rightarrow$ $\rightarrow 0$. The uniformly convex spaces are reflexive and have another important property: any bounded subset $E \subset X$ is weakly compact. Moreover, if a sequence $\left\{x_{n}\right\} \subset X$ is weakly convergent to $x_{0}$ and $\left\|x_{n}\right\| \rightarrow\left\|x_{0}\right\|$, then $x_{n} \rightarrow x_{0}$ in strong topology of the space $X$ induced by its norm. All this is valid, for example, for any Hilbert space and for $L_{p}$ spaces with $p>1$.

2. As for the finite dimensional case, we can show that the answer is negative for the spaces $\mathbf{T}(0, n)$ of the punctured spheres (the surfaces of genus zero). Let

$$
\mathbb{C}_{\mathbf{a}}=\widehat{\mathbb{C}} \backslash\left\{a_{1}, \ldots, a_{n}\right\}, \quad \widehat{\mathbb{C}}=\mathbb{C} \cup\{\infty\},
$$

where $\mathbf{a}=\left(a_{1}, \ldots, a_{n}\right)$ is an ordered collection of $n>4$ deleted distinct points. Note that $\operatorname{dim} \mathbf{T}(0, n)=n-3$ and that the one-dimensional space $\mathbf{T}(0,4)$ is conformally equivalent to the disk.

Theorem 2. There is an integer $n_{0}>4$ such that any space $\mathbf{T}(0, n)$ with $n \geq n_{0}$ cannot be mapped biholomorphically onto a bounded convex domain in $\mathbb{C}^{n-3}$.

The proof of this theorem also involves conformally rigid domains (as for all results mentioned above) and an important interpolation theorem for bounded univalent functions in the plane domains. This approach also has other interesting applications that are not presented here.

3. First we recall some needed facts from the Teichmüller space theory. Consider the ordered $n$-tuples of points

$$
\mathbf{a}=\left(1, i,-1, a_{1}, \ldots, a_{n-3}\right), \quad n>4,
$$


with distinct $a_{j} \in \widehat{\mathbb{C}} \backslash\{1, i,-1\}$ and the corresponding punctured spheres

$$
X_{\mathbf{a}}=\widehat{\mathbb{C}} \backslash\left\{1, i,-1, a_{1}, \ldots, a_{n-3}\right\}, \quad \widehat{\mathbb{C}}=\mathbb{C} \cup\{\infty\},
$$

regarded as the Riemann surfaces of genus zero. Fix a collection

$$
\mathbf{a}^{0}=\left(1, i,-1, a_{1}^{0}, \ldots, a_{n-3}^{0}\right)
$$

defining the base point $X_{\mathbf{a}^{0}}$ of Teichmüller space $\mathbf{T}(0, n)=\mathbf{T}\left(X_{\mathbf{a}^{0}}\right)$. Its points are the equivalence classes $[\mu]$ of Beltrami coefficients from the ball

$$
\operatorname{Belt}(\mathbb{C})_{1}=\left\{\mu \in L_{\infty}(\mathbb{C}):\|\mu\|_{\infty}<1\right\},
$$

under the relation that $\mu_{1} \sim \mu_{2}$ if the corresponding quasiconformal homeomorphisms $w^{\mu_{1}}, w^{\mu_{2}}: X_{\mathbf{a}^{0}} \rightarrow X_{\mathbf{a}}$ (the solutions of the Beltrami equation $\bar{\partial} w=\mu \partial w$ with $\mu=\mu_{1}, \mu_{2}$ ) are homotopic on $X_{\mathbf{a}^{0}}$ (and hence coincide in the points $1, i,-1, a_{1}^{0}, \ldots, a_{n-3}^{0}$ ). This models $\mathbf{T}(0, n)$ as the quotient space $\mathbf{T}(0, n)=\operatorname{Belt}(\mathbb{C})_{1} / \sim$ with complex Banach structure of dimension $n-3$ inherited from the ball $\operatorname{Belt}(\mathbb{C})_{1}$. Note that $\mathbf{T}(0, n)$ is a complete metric space with intrinsic Teichmüller metric defined by quasiconformal maps. By Royden's theorem, this metric equals the Kobayashi metric determined by the complex structure.

Another canonical model of $\mathbf{T}(0, n)=\mathbf{T}\left(X_{\mathbf{a}^{0}}\right)$ is obtained using the uniformization of Riemann surfaces and the holomorphic Bers embedding of Teichmüller spaces. We now consider the disks

$$
\Delta=\{z:|z|<1\}, \quad \Delta^{*}=\{z \in \widehat{\mathbb{C}}:|z|>1\}
$$

and the ball of Beltrami coefficients (conformal structures on $\mathbb{D}$ )

$$
\operatorname{Belt}(\Delta)_{1}=\left\{\mu \in L_{\infty}(\mathbb{C}): \mu \mid \Delta^{*}=0,\|\mu\|_{\infty}<1\right\} .
$$

and model the universal Teichmüller space $\mathbf{T}=\mathbf{T}(\mathbb{D})$ as the space of quasi-symmetric homeomorphisms of the unit circle $S^{1}=\partial \Delta$ factorized by Möbius maps. The canonical complex Banach structure on $\mathbf{T}$ is defined by factorization of this ball, letting $\mu, v \in \operatorname{Belt}(\Delta)_{1}$ be equivalent if the corresponding quasiconformal maps $w^{\mu}, / w^{v}$ of $\widehat{\mathbb{C}}$ coincide on the circle $S^{1}$ and passing to their Schwarzian derivatives $S_{w^{\mu}}(z)$ in $D^{*}$ now running over a bounded domain in the space $\mathbf{B}=\mathbf{B}\left(\Delta^{*}\right)$ of holomorphic functions $\varphi$ in $\Delta^{*}$ with norm $\|\varphi\|=\sup _{\mathbb{D}^{*}}\left(|z|^{2}-1\right)^{2}|\varphi(z)|$. This domain is contained in the ball $\left\{\|\varphi\|_{\mathbf{B}}<1 / 6\right\}$.

The map $\phi: \mu \rightarrow S_{w^{\mu}}$ is holomorphic and descends to a biholomorphic map of the space $\mathbf{T}$ onto this domain, which we will identify with $\mathbf{T}$. It contains as complex submanifolds the Teichmüller spaces of all hyperbolic Riemann surfaces and of Fuchsian groups.

As is well-known, the space $\mathbf{T}$ coincides with the union of inner points of the set

$$
\mathbf{S}=\left\{\varphi=S_{w} \in \mathbf{B}: w \text { univalent in } \Delta^{*}\right\}
$$

on the other hand, by Thurston's theorem, $\mathbf{S} \backslash \overline{\mathbf{T}}$ has uncountable many isolated points $\varphi_{0}=S_{w_{0}}$ which correspond to conformally rigid domains $w_{0}\left(\Delta^{*}\right)$.

4. Using the holomorphic universal covering map $h: \Delta \rightarrow X_{\mathbf{a}^{0}}$, one represents the surface $X_{\mathbf{a}^{0}}$ as the quotient space $\Delta / \Gamma_{0}$ (up to conformal equivalence), where $\Gamma_{0}$ is a torsion free Fuchsian group of the first kind acting discontinuously on $\Delta \cup \Delta^{*}$. The functions 
$\mu \in L_{\infty}\left(X_{\mathbf{a}^{0}}\right)$ are lifted to $U$ as the Beltrami $(-1,1)$-measurable forms $\tilde{\mu} d \bar{z} / d z$ in $\Delta$ with respect to $\Gamma_{0}$ which satisfy $(\widetilde{\mu} \circ \gamma) \overline{\gamma^{\prime}} / \gamma^{\prime}=\widetilde{\mu}, \gamma \in \Gamma_{0}$ and form the Banach space $L_{\infty}\left(\Delta, \Gamma_{0}\right)$.

We extend these $\widetilde{\mu}$ by zero to $U^{*}$ and consider the unit ball $\operatorname{Belt}\left(\Delta, \Gamma_{0}\right)_{1}$ of $L_{\infty}\left(\Delta, \Gamma_{0}\right)$. Then the corresponding Schwarzians $S_{w^{\widetilde{\mu}} \mid \Delta^{*}}$ belong to the universal Teichmüller space $\mathbf{T}$ and the subspace of such Schwarzians is regarded as the Teichmüller space $\mathbf{T}\left(\Gamma_{0}\right)$ of the group $\Gamma_{0}$. It is canonically isomorphic to the space $\mathbf{T}\left(X_{\mathbf{a}^{0}}\right)$. Moreover,

$$
\mathbf{T}\left(\Gamma_{0}\right)=\mathbf{T} \cap \mathbf{B}\left(\Gamma_{0}\right),
$$

where $\mathbf{B}\left(\Gamma_{0}\right)$ is an $(n-3)$-dimensional subspace of $\mathbf{B}$ which consists of elements $\varphi \in \mathbf{B}$ satisfying $(\varphi \circ \gamma)\left(\gamma^{\prime}\right)^{2}=\varphi$ for all $\gamma \in \Gamma_{0}$; see, e.g., [13]. This leads to the representation of the space $\mathbf{T}\left(X_{\mathbf{a}^{0}}\right)$ as a bounded domain in the complex Euclidean space $\mathbb{C}^{n-3}$.

Note also that the space $\mathbf{B}$ is dual to the subspace $A_{1}\left(\Delta^{*}\right)$ in $L_{1}\left(\Delta^{*}\right)$ formed by integrable holomorphic functions in $\Delta^{*}$, while $B\left(\Delta^{*}, \Gamma_{0}\right)$ has the same elements as the space $A_{1}\left(\Delta^{*}, \Gamma_{0}\right)$ of integrable holomorphic forms of degree -4 with norm $\|\varphi\|=\iint_{\Delta^{*} / \Gamma_{0}}|\varphi(z)| d x d y$.

5. The collections (5) fill a domain $\mathcal{U}_{n}$ in $\mathbb{C}^{n-3}$ obtained by deleting from this space the hyperplanes $\left\{z=\left(z_{1}, \ldots, z_{n-3}\right): z_{j}=z_{l}, j \neq l\right\}$, and with $z_{1}=1, z_{2}=i, z_{3}=-1$. This domain represents the Torelli space of the spheres $X_{\mathbf{a}}$ and is covered by $\mathbf{T}(0, n)$. Namely, we have (see, e.g., [14, Section 2.8])

Lemma 1. The holomorphic universal covering space of $\mathcal{U}_{n}$ is the Teichmüller space $\mathbf{T}(0, n)$. This means that for each punctured sphere $X_{\mathbf{a}}$, there is a holomorphic universal covering

$$
\pi_{\mathbf{a}}: \mathbf{T}(0, n)=\mathbf{T}\left(X_{\mathbf{a}}\right) \rightarrow \mathcal{U}_{n} .
$$

The covering map $\pi_{a}$ is well defined by

$$
\pi_{\mathbf{a}} \circ \phi_{\mathbf{a}}(\mu)=\left(1, i,-1, w^{\mu}\left(a_{1}\right), \ldots, w^{\mu}\left(a_{n-3}\right)\right),
$$

where $\phi_{\mathbf{a}}$ denotes the canonical projection of the ball $\operatorname{Belt}(\Delta)_{1}$ onto the space $\mathbf{T}\left(X_{\mathbf{a}}\right)$.

Truncated collections $\mathbf{a}_{*}=\left(a_{1}, \ldots, a_{n-3}\right)$ provide the local complex coordinates on the space $\mathbf{T}(0, n)$ and define its complex structure.

Let us consider the ball $\operatorname{Belt}(\Delta)_{1}$ and call its elements $\mu$ defining the same point of the universal Teichmüller space T-equivalent. The corresponding homeomorphisms $w^{\mu}$ coincide on the unit circle.

We now assume that the coordinates $a_{j}^{0}$ of the surface $X_{\mathbf{a}^{0}}$ are placed on the circle $S^{1}$ and define on this ball another equivalence relation, letting $\mu, v \in \operatorname{Belt}(\Delta)_{1}$ be equivalent if $w^{\mu}\left(a_{j}^{0}\right)=w^{v}\left(a_{j}^{0}\right)$ for all $j$ and the homeomorphisms $w^{\mu}, w^{v}$ are homotopic on the punctured sphere $X_{\mathbf{a}^{0}}$. Let us call such $\mu$ and $v$ strongly $n$-equivalent. This equivalence is weaker than $\mathbf{T}$-equivalence, i.e., if two coefficients $\mu, v \in \operatorname{Belt}(\Delta)_{1}$ are $\mathbf{T}$-equivalent, then they are also strongly $n$-equivalent, which implies, (by descending to the equivalence classes) a holomorphic map $\chi$ of the underlying space $\mathbf{T}$ into $\mathbf{T}(0, n)=\mathbf{T}\left(X_{\mathbf{a}^{0}}\right)$.

This map is a split immersion, i.e., it has local holomorphic sections. In fact, we have much more:

Lemma 2. The map $\chi$ is surjective and has a global holomorphic section $s: \mathbf{T}\left(X_{\mathbf{a}^{\mathbf{0}}}\right) \rightarrow \mathbf{T}$.

Proof. The surjectivity of $\chi$ is a consequence of the following interpolation result from [5]. 
Lemma 3. Given two cyclically ordered collections of points $\left(z_{1}, \ldots, z_{m}\right)$ and $\left(\zeta_{1}, \ldots, \zeta_{m}\right)$ on the unit circle $S^{1}=\{|z|=1\}$, there exists a holomorphic univalent function $f$ in the closure of the unit disk $\Delta=\{|z|<1\}$ such that $|f(z)|<1$ for $z \in \bar{\Delta}$ distinct from $z_{1}, \ldots, z_{m}$, and $f\left(z_{k}\right)=\zeta_{k}$ for all $k=1, \ldots, m$. Moreover, there exist univalent polynomials $f$ with such an interpolation property.

Since the interpolating function $f$ given by this lemma is regular up to the boundary, it can be extended quasiconformally across the boundary circle $S^{1}$ to the whole sphere $\widehat{\mathbb{C}}$. Hence, given a cyclically ordered collection $\left(z_{1}, \ldots, z_{m}\right)$ of points on $S^{1}$, then for any ordered collection $\left(\zeta_{1}, \ldots, \zeta_{m}\right)$ in $\widehat{\mathbb{C}}$, there is a quasi-conformal homeomorphism $\widehat{f}$ of the whole sphere $\widehat{\mathbb{C}}$ carrying the points $z_{j}$ to $\zeta_{j}, j=1, \ldots, m$, and such that its restriction to the closed disk $\bar{\Delta}$ is biholomorphic on $\bar{\Delta}$ (and similarly for the ordered collections of points on arbitrary quasicircles).

Applying Lemma 1, one constructs quasiconformal extensions of $f$ lying in prescribed homotopy classes of homeomorphisms $X_{\mathbf{z}} \rightarrow X_{\mathrm{w}}$.

To prove the assertion of Lemma 2 on holomorphic sections for $\chi$, take a dense subset

$$
e=\left\{x_{1}, x_{2}, \ldots\right\} \subset X_{\mathbf{a}^{0}} \cap S^{1}
$$

accumulating to all points of $S^{1}$ and consider the surfaces

$$
X_{\mathbf{a}^{0}}^{m}=X_{\mathbf{a}^{0}} \backslash\left\{x_{1}, \ldots, x_{m}\right\}, \quad m \geq 1
$$

(having type $(0, n+m))$. The equivalence relations on $\operatorname{Belt}(\mathbb{C})_{1}$ for $X_{\mathbf{a}^{0}}^{m}$ and $X_{\mathbf{a}^{0}}$ generate a holomorphic map $\chi_{m}: \mathbf{T}\left(X_{\mathbf{a}^{0}}^{m}\right) \rightarrow \mathbf{T}\left(X_{\mathbf{a}^{0}}\right)$.

The inclusion map $j_{m}: X_{\mathbf{a}^{0}}^{m} \hookrightarrow X_{\mathbf{a}^{0}}$ forgetting the additional punctures generates a holomorphic embedding $s_{m}: \mathbf{T}\left(X_{\mathbf{a}^{0}}\right) \hookrightarrow \mathbf{T}\left(X_{\mathbf{a}^{0}}^{m}\right)$ inverting $\chi_{m}$. To present this section analytically, we uniformize the surface $X_{\mathrm{a}^{0}}^{m}$ by a torsion free Fuchsian group $\Gamma_{0}^{m}$ on $\Delta \cup \Delta^{*}$ so that $X_{\mathbf{a}^{0}}^{m}=\Delta / \Gamma_{0}^{m}$. By (6), its Teichmüller space $\mathbf{T}\left(\Gamma_{0}^{m}\right)=\mathbf{T} \cap \mathbf{B}\left(\Gamma_{0}^{m}\right)$.

The holomorphic universal covering maps $h: \Delta^{*} \rightarrow \Delta^{*} / \Gamma_{0}$ and $h^{m}: \Delta^{*} \rightarrow \Delta^{*} / \Gamma_{0}^{m}$ are related by $j \circ h^{m}=h \circ \widehat{j}$, where $\widehat{j}$ is the lift of $j$. This induces a surjective homomorphism of the covering groups $\theta_{m}: \Gamma_{0}^{m} \rightarrow \Gamma_{0}$ by

$$
\widehat{j} \circ \gamma=\theta_{m}(\gamma) \circ \gamma, \quad \gamma \in \Gamma_{0}^{m},
$$

and the norm preserving isomorphism $\widehat{j}_{m, *}: \mathbf{B}\left(\Gamma_{0}\right) \rightarrow \mathbf{B}\left(\Gamma_{0}^{m}\right)$ by

$$
\widehat{j}_{m, *} \varphi=(\varphi \circ \widehat{j})\left(\hat{j}^{\prime}\right)^{2},
$$

which projects to the surfaces $X_{\mathbf{a}^{0}}$ and $X_{\mathbf{a}^{0}}^{m}$ as the inclusion of the space $Q\left(X_{\mathbf{a}^{0}}\right)$ of quadratic differentials corresponding to $\mathbf{B}\left(\Gamma_{0}\right)$ into the space $Q\left(X_{\mathbf{a}^{0}}^{m}\right)$ (cf. [6]). The equality (8) represents the section $s_{m}$ indicated above.

6. To investigate the limit function for $m \rightarrow \infty$, we embed $\mathbf{T}$ into the space $\mathbf{B}$ and compose each $s_{m}$ with a biholomorphism

$$
\eta_{m}: \mathbf{T}\left(X_{\mathbf{a}^{0}}^{m}\right) \rightarrow \mathbf{T}\left(\Gamma_{0}^{m}\right)=\mathbf{T} \cap \mathbf{B}\left(\Gamma_{0}^{m}\right) \quad(m=1,2, \ldots) .
$$

Then the elements of $\mathbf{T}\left(\Gamma_{0}^{m}\right)$ are represented in the form

$$
\widehat{s}_{m}(z, \cdot)=S_{f^{m}}\left(z ; X_{\mathbf{a}}\right),
$$


being parameterized by the points of $\mathbf{T}\left(X_{\mathbf{a}^{0}}\right)$.

Each $\Gamma_{0}^{m}$ is the covering group of the universal cover $h_{m}: \Delta * \rightarrow X_{\mathbf{a}_{0}^{m}}$, which can be normalized (conjugating appropriately $\Gamma_{0}^{m}$ ) by $h_{m}(\infty)=\infty, h_{m}^{\prime}(\infty)>0$. Take its fundamental polygon $P_{m}$ obtained as the union of the regular circular $m$-gon in $\Delta^{*}$ centered at the infinite point with the zero angles at the vertices and its reflection with respect to one of the boundary arcs. These polygons increasingly exhaust the disk $\Delta^{*}$ from inside; hence, by the Carathéodory kernel theorem, the maps $h_{m}$ converge to the identity map locally uniformly in $\Delta^{*}$.

Since the set of punctures $e$ is dense, it completely determines the equivalence classes $\left[w^{\mu}\right]$ and $S_{w^{\mu}}$ of $\mathbf{T}$, and the limit function $s(z, \cdot)=\lim _{m \rightarrow \infty} \widehat{s}_{m}(z, \cdot)$ maps $\mathbf{T}\left(X_{\mathbf{a}^{0}}\right)$ into $\mathbf{T}$. For any fixed $X_{\mathbf{a}}$, this function is holomorphic on $\Delta^{*}$; hence, by the well-known property of elements in the functional spaces with sup-norms, $s(z, \cdot)$ is holomorphic also in the norm of $\mathbf{B}$. This $s$ determines a holomorphic section of the original map $\chi$, which completes the proof of Lemma 3.

7. The following lemma is a special case of the general approximation lemma in [12]; it reveals some special features which are used also in the proof of Theorem 2 .

Lemma 4. For any Schwarzian $\varphi \in \mathbf{T}$ holomorphic in the disk $\Delta_{r}^{*}=\{|z|>r\}, r<1$, there exist a sequence of torsion free Fuchsian groups $\Gamma_{m}^{r}$ of the first kind acting on $\Delta_{r}^{*}$, which does not depend on $\varphi$, and a sequence of elements $\varphi_{m} \in \mathbf{T}\left(\Gamma_{m}^{r}\right)$ canonically determined by $\varphi$ and converging to $\varphi$ uniformly on $\overline{\Delta^{*}}$; hence, $\lim _{m \rightarrow \infty}\left\|\varphi_{m}-\varphi\right\|_{\mathbf{B}}=0$.

Proof. We pass to maps $w^{\mu}$ preserving the points $0,1, \infty$ (which does not affect their Schwarzians $S_{w^{\mu}}$ forming the space $\mathbf{T}$ ) and pick on the unit circle $S^{1}$ a dense subset of dyadic points

$$
a_{l}^{(n)}=e^{\pi l i / 2^{m}} ; l=0,1, \ldots, 2^{m+1}-1 ; m=2,3, \ldots .
$$

Regarding the collections

$$
\mathbf{a}^{0}(r, m)=\left\{0, r, r e^{\pi l i / 2^{m-3}}, \infty ; l=0,1, \ldots, m-1\right\}
$$

as the punctures of the base points $X_{\mathbf{a}^{0}(r, n)}$ of the spaces $\mathbf{T}(0, m)=\mathbf{T}\left(X_{\mathbf{a}^{0}(r, m)}\right)$, consider for each $m$ the covering group $\Gamma_{m}^{r}$ of the universal cover $h_{m}: \Delta_{r}^{*} \rightarrow X_{\mathbf{a}_{0}^{(r, m)}}$ with $h_{m}(\infty)=$ $=\infty, h_{m}^{\prime}(\infty)>0$ and take its canonical fundamental polygon $P_{m}$ in $\Delta_{r}^{*}$ centered at the infinite point with the zero angles at the vertices. These polygons increasingly exhaust the disk $\Delta_{r}^{*}$ from inside, hence the maps $h_{m}$ converge to the identity map locally uniformly in $\Delta_{r}^{*}$.

The classical result of geometric function theory implies that for each non-zero $\varphi \in$ $\in \mathbf{B}\left(\Delta_{r}^{*}\right)$ and large $m \geq m_{0}(\varphi)$, the corresponding $\Gamma_{m}^{r}$-quadratic differentials

$$
\varphi_{m}(z)=\sum_{\gamma \in \Gamma_{m}^{r}} \varphi(\gamma z) \gamma^{\prime}(z)^{2}
$$

also do not vanish and are the Schwarzians of univalent functions $w_{m}$ on $\Delta_{r}^{*}$ compatible with these groups. The sequences $\left\{\Gamma_{m}^{r}\right\}$ and $\left\{\varphi_{n}\right\}$ satisfy the assertion of the lemma.

Now, to complete the proof of Theorem 2, assume, to the contrary, that there exists an infinite sequence of spaces $\mathbf{T}(0, n)$ admitting biholomorphic homeomorphisms $\eta_{n}$ onto the bounded convex domains $D_{n} \subset \mathbb{C}^{n-3}$, where $n$ runs over an infinite subsequence from $\mathbb{N}$. 
We embed these domains $D_{n}$ biholomorphically as convex submanifolds $V_{n}$ into the unit ball $B\left(l^{2}\right)$ of the Hilbert space $l^{2}$ of sequences so that each $V_{n}$ is placed in $n$-3-dimensional subspace $l_{n}^{2}$ of $l^{2}$ formed by points $\mathbf{c}=\left(c_{j}\right)$ with $c_{j}=0$ for all $j>n$ and contains its origin, $V_{n} \subset V_{n+1}$, and $V_{n}$ touches $V_{n+1}$ from inside in its boundary point $\mathbf{c}_{n} \in \partial V_{n}$ whose distance from the origin is maximal. Their union

$$
V_{\infty}=\bigcup_{n} V_{n}
$$

is a convex submanifold in the ball $B\left(l^{2}\right)$ whose completion $\widehat{V}_{\infty}$ is a convex domain $\widehat{V}_{\infty}$ in a subspace $l_{0}^{2}$ of $l^{2}$.

Now take a Schwarzian $\varphi_{*}=S_{w^{*}} \in \mathbf{S} \backslash \mathbf{T}$ defining an isolated point of $\mathbf{S}$ (hence a conformally rigid domain $w^{*}\left(\Delta^{*}\right)$ in $\left.\widehat{\mathbb{C}}\right)$ and consider the homotopy functions $w_{t}^{*}(z)=$ $=t w^{*}(z / t)$. Each $w_{t}^{*}$ is conformal in the wider disk $\Delta_{1 /|t|}$. Pick a sequence of positive numbers $t_{j}$ approaching 1 and apply Lemma 6 to approximate each Schwarzian

$$
\psi_{j}(z):=S_{w_{t_{j}}^{*}}(z)=t_{j}^{-2} S_{w^{*}}\left(z / t_{j}\right)
$$

by differentials $\varphi_{m_{j}} \in \mathbf{T}\left(\Gamma_{m}^{t_{j}}\right)$ satisfying

$$
\left\|\varphi_{m_{j}}-\psi_{j}\right\|_{\mathbf{B}}<\frac{1}{2^{j}} \operatorname{dist}\left(\psi_{j}, \partial \mathbf{T}\right) .
$$

These $\varphi_{m_{j}}$ are determined by $\psi_{j}$ (hence by original $\varphi_{*}$ ) via (9) and are convergent to $\varphi_{*}$ locally uniformly in $\Delta^{*}$.

Moreover, the proof of Lemma 6 shows that one can choose in the series (9) a sufficiently large number $m_{j}=n$ so that $\mathbf{T}\left(\Gamma_{m}^{t_{j}}\right)=\mathbf{T}\left(\Gamma_{n}\right)$ is one of the spaces listed above equivalent to convex domains $V_{n}$.

We have for each $n$ commutative diagram

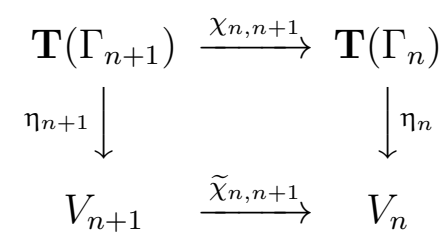

where $\chi_{n, n+1}$ is again a holomorphic map generated by forgetting the additional puncture on the base point of $\mathbf{T}(0, n)$ and $\widetilde{\chi}_{n, n+1}=\eta_{n}^{-1} \circ \chi_{n, n+1} \circ \eta_{n+1}$. We can replace in (10) each domain $V_{n}$ by its image $\widetilde{\chi}_{n, n+1}^{-1}\left(V_{n}\right)$ in $V_{n+1}$.

Denote by $\chi_{n}$ the holomorphic map $\mathbf{T} \rightarrow \mathbf{T}_{n}$ given by Lemma 3. Its composition with $\eta_{n}$ tends as $n \rightarrow \infty$ to a holomorphic map

$$
\eta_{\infty}=\lim _{n \rightarrow \infty} \eta_{n} \circ \chi_{n}: \mathbf{T} \rightarrow V_{\infty}
$$

Its holomorphy is ensured by the infinite dimensional analog of Montel's theorem following from the Alaoglu - Bourbaki theorem.

It follows from Lemma 3 that $\eta_{\infty}$ has a holomorphic section $\sigma_{\infty}: V_{\infty} \rightarrow \mathbf{T}$ mapping $V_{\infty}$ biholomorphically onto a domain

$$
\mathbf{T}_{\infty}=\bigcup_{n} \chi_{n, n+1}^{-1} \mathbf{T}\left(\Gamma_{n}\right) \subset \mathbf{T} \cap \mathbf{B}_{0}
$$


where $\mathbf{B}_{0}$ is some subspace of $\mathbf{B}$ (cf. (6)). Its inverse $\eta_{\infty}^{-1}$ also is holomorphic.

Noting that the sequence of images $\mathbf{x}_{n}=\eta_{\infty}\left(\varphi_{n}\right) \in V_{\infty}$ is weakly compact in the space $l^{2}$ and passing if needed to a convergent subsequence to some point $\mathbf{x}_{0} \in l^{2}$, one gets

$$
\left\|\mathbf{x}_{0}\right\|_{l^{2}} \leq \lim _{n \rightarrow \infty}\left\|\mathbf{x}_{n}\right\|_{l^{2}}
$$

Our goal is to show that only the equality is possible here, i.e., $\left\|\mathbf{x}_{0}\right\|_{l^{2}}=\lim _{n \rightarrow \infty}\left\|\mathbf{x}_{n}\right\|_{l^{2}}$. To this end, we consider the space $l_{0}^{2}$ as a real space with the same norm (admitting multiplication of $\mathbf{x} \in l_{0}^{2}$ only with $c \in \mathbb{R}$ ). Denote this real space by $\widetilde{l}_{0}^{2}$. The domain $V_{\infty}$ is convex in $\widetilde{l}_{0}^{2}$; thus its Minkowski functional

$$
\alpha(\mathbf{x})=\inf \left\{t>0: t^{-1} \mathbf{x} \in V_{\infty}\right\} \quad\left(\mathbf{x} \in \widetilde{l}_{0}^{2}\right)
$$

determines on this space a norm equivalent to initial norm $\|\mathbf{x}\|_{l^{2}}$. Denote the space with the new norm by $\widetilde{l}_{\alpha}^{2}$ and notice that the domain $V_{\infty}$ is its unit ball.

The sequence $x_{n}$ is weakly convergent also on $\widetilde{l}_{\alpha}^{2}$; thus, similar to (11),

$$
\alpha\left(\mathbf{x}_{0}\right) \leq \lim _{n \rightarrow \infty} \alpha\left(\mathbf{x}_{n}\right) \leq 1
$$

This implies that the point $\mathbf{x}_{0}$ belongs to the closure of the domain $V_{\infty}$ in $l^{2}$-norm.

If $\alpha\left(\mathbf{x}_{0}\right)<\lim _{n \rightarrow \infty} \alpha\left(\mathbf{x}_{n}\right)$ or $\alpha\left(\mathbf{x}_{0}\right)=\lim _{n \rightarrow \infty} \alpha\left(\mathbf{x}_{n}\right)<1$, in both these cases the point $\mathbf{x}_{0}$ must lie inside $V_{\infty}$. Then its inverse image $\eta^{-1}\left(\mathbf{x}_{0}\right) \in \mathbf{T}$ and thus is the Schwarzian $S_{w_{0}}$ of some univalent function $w_{0}$ on $\Delta^{*}$. Since $\eta^{-1}\left(\mathbf{x}_{n}\right)=\varphi_{n}$ are convergent locally uniformly on $\Delta^{*}$ to $S_{w^{*}}$, it must be $w_{0}=w^{*}$ which yields that $S_{w^{*}}$ must lie in $\mathbf{T}_{\infty} \subset \mathbf{T}$, in contradiction to that it is an isolated point of the set $\mathbf{S}$.

It remains the case $\alpha\left(\mathbf{x}_{0}\right)=\lim _{n \rightarrow \infty} \alpha\left(\mathbf{x}_{n}\right)=1$ which is equivalent to

$$
\lim _{n \rightarrow \infty}\left\|\mathbf{x}_{n}\right\|_{l^{2}}=\left\|\mathbf{x}_{0}\right\|_{l^{2}} \quad \text { and } \quad \mathbf{x}_{0} \in \partial V_{\infty}
$$

The weak convergence $\mathbf{x}_{n} \rightarrow \mathbf{x}_{0}$ in $l^{2}$ and the equality (12) together imply the strong convergence $\lim _{n \rightarrow \infty}\left\|\mathbf{x}_{n}-\mathbf{x}_{0}\right\|_{l^{2}}=0$.

Then, since $\eta_{\infty}$ is a biholomorphic homeomorphism, the inverse images $\eta_{\infty}^{-1}\left(\mathbf{x}_{n}\right)=\varphi_{n}$ must approach the boundary of $\mathbf{T}$ in $\mathbf{B}$ and therefore $S_{w *}$ must be a boundary point of $\mathbf{T}$, again contradicting that it is an isolated point of $\mathbf{S}$. This completes the proof of the theorem.

\section{REFERENCES}

1. Abikoff W. A geometric property of Bers' embedding of the Teichmüller space. Riemann Surfaces and Related Topics (AM-97): Proceedings of the 1978 Stony Brook Conference (Ann. of Math. Stud.). Princeton, N. J., Princeton University Press, 1981, pp. 3-5.

2. Astala K. Selfsimilar zippers. Holomorphic Functions and Moduli. New York, Springer, 1988, vol. I, pp. 61-73.

3. Bers L., Kra I. A Crash Course on Kleinian Groups. Lecture Notes in Mathematics. Berlin, Springer, 1974, vol. 400, pp. 1-134.

4. Bers L. On a theorem of Abikoff. Ann. Acad. Sci. Fenn. Ser. A. I. Math., 1985, vol. 10, pp. 83-87.

5. Clunie J.G., Hallenbeck D.J., MacGregor T.H. A peaking and interpolation problem for univalent functions. J. Math. Anal. Appl., 1985, vol. 111, pp. 559-570. 
6. Earle C.J., Kra I. On sections of some holomorphic families of closed Riemann surfaces. Acta Math., 1976, vol. 137, pp. 49-79.

7. Krushkal S.L. On shape of Hilbertian embedding of universal Teichmüller space. Transactions of Inst. of Math. Nat. Acad. Sciences of Ukraine (Zb. Pr. Inst. Mat. NAN Ukraine), 2015, vol. 12, no. 3, pp. 160-163.

8. Krushkal S.L. On shape of Teichmüller spaces. Journal of Analysis, 2014, vol. 22, pp. 69-76.

9. Krushkal S.L. On the question of the structure of the universal Teichmüller space. Soviet Math. Dokl., 1989, vol. 38, pp. 435-437.

10. Krushkal S.L. Quasiconformal Mappings and Riemann Surfaces. New York, Wiley, 1979. 332 p.

11. Krushkal S.L. Schwarzian derivative and complex Finsler metrics. Contemporary Mathematics, 2005, vol. 382, pp. 243-262.

12. Krushkal S.L. Teichmüller spaces are not starlike. Ann. Acad. Sci. Fenn. Ser. A. I. Math., 1995, vol. 20, pp. 167-173.

13. Lehto O. Univalent Functions and Teichmüller Spaces. New York, Springer-Verlag, 1987. $260 \mathrm{p}$. $427 \mathrm{p}$

14. Nag S. The Complex Analytic Theory of Teichmüller Spaces. New York, Wiley, 1988.

15. Thurston W.P. Zippers and univalent functions. The Bieberbach Conjecture: Proceedings of the Symposium on the Occasion of its Proof. Providence, R. I., Amer. Math. Soc., 1986, pp. 185-197.

16. Toki M. On non-starlikeness of Teichmüller spaces. Proc. Japan Acad. Ser. A, 1993, vol. 69, pp. 58-60. 142.

17. Tukia P. The space of quasisymmetric mappings. Math. Scand., 1977, vol. 40, pp. 127-

18. Zhuravlev I.V. A topological property of Teichmüller space. Math. Notes, 1985, vol. 38, pp. 803-804.

\section{КОМПЛЕКСНАЯ ЖЕСТКОСТЬ ПРОСТРАНСТВ ТЕЙХМЮЛЛЕРА}

\section{Самуил Лейбович Крушкаль}

Доктор физико-математических наук, профессор факультета математики,

Bar-Ilan University, Ramat-Gan, 5290002, Israel,

University of Virginia, Charlottesville, VA 22904-4137, USA

slk6z@cms.mail.virginia.edu

Аннотация. В работе представлены старые и новые результаты относительно хорошо известных задач в теории пространств Тейхмюллера. А именно: являются ли эти пространства звездообразными в голоморфном вложении Берса, и существуют ли пространства Тейхмюллера размерности больше 1 биголоморфно эквивалентные ограниченной выпуклой области в комплексном банаховом пространстве.

Ключевые слова: пространства Тейхмюллера, голоморфное вложение, производная Шварца, выпуклая область, звездообразность, голоморфное сечение, конформно жесткая область, равномерно выпуклое банахово пространство. 